Pure and Applied Mathematics Quarterly

Volume 7, Number 3

(Special Issue: In honor of

Jacques Tits)

$969-994,2011$

\title{
Remarks on Parabolic Character Sheaves
}

\author{
T. A. Springer
}

Dedicated to Jacques Tits on the occasion of his eightieth birthday

\begin{abstract}
The paper contains comments on the parabolic character sheaves associated to a connected reductive group $G$, introduced by Lusztig in [L2]. A correspondence is established between parabolic character sheaves and certain perverse sheaves on $G$, equivariant under a subgroup $\Delta$ of $G \times G$, acting on $G$ in the usual way (see Th. 3.8). Section 6 is about comments on results of He in [H3].
\end{abstract}

Keywords: parabolic character sheaves, $\Delta$ - character sheaves

\section{Introduction.}

0.1. The present paper arose out of an attempt to get some understanding of Lusztig's parabolic character sheaves, introduced by him in [L2].

Let $G$ be a connected reductive group. Parabolic character sheaves are $G$ equivariant irreducible perverse sheaves on certain $G$-varieties $Z$, of the same dimension as $G$. Lusztig's original character sheaves are obtained for $Z=G$.

If $G$ is adjoint there is a particular case, where $Z$ is a torus bundle over a variety $\bar{Z}$ which appears in the wonderful compactification $X$ of $G$. Namely, $X$ is a variety on which $G \times G$ acts with finitely many orbits and $\bar{Z}$ is one of them. Some

Received June 3, 2009 
parabolic character sheaves will lead to irreducible perverse sheaves on $G \times G$ orbits in $X$ that extend canonically to $X$. They are equivariant for the action of the diagonal subgroup $G_{d}$ of $G \times G$. The extension should be viewed as a character sheaf on $X$.

0.2. A rather obvious outgrowth of [MS1] and [Sp1] is another definition of character sheaves on $X$. I convinced myself, by an argument using the Hecke algebra representation of [Sp1] that the character sheaves on $X$ so obtained coincide with Lusztig's.

Independently, Xuhua He defined character sheaves on $X$ in the same manner as I did. He proved the coincidence with Lusztig's character sheaves in [H3], more or less by direct computation.

The Hecke algebra aspects are discussed in Section 4 of this paper (more generally, for $Z$ ). The relation with compactifications is briefly discussed in Section 5 .

0.3. A main result of Lusztig's work is another description of parabolic character sheaves, using a decomposition of $Z$ into " $G$-stable pieces". The decomposition is also useful in other contexts, as is shown in He's work (see e.g. [H1, H2]). Lusztig also establishes a connection between parabolic character sheaves and character sheaves on disconnected groups.

In Lusztig's analysis an important role is played by a combinatorial machinery due to Bédard. This is quite involved (anyway, in my view). In trying to get a grip on it I noticed that the machinery can be broken into pieces.

In each piece there is the following situation. One has two algebraic groups $G$ and $H$ and a $G \times H$-variety $X$. Assume that quotients (i.e. orbit spaces) $G \backslash X$ and $H \backslash X$ exists. Then there is a strong connection between the $G$-action on $H \backslash X$ and the $H$-action on $G \backslash X$. For example, there is a closure preserving bijection between orbits and an equivalence of categories of equivariant perverse sheaves. Establishing such results is quite easy, see Section 1. It turns out that they are useful in understanding Lusztig's analysis.

0.4. The results of Section 1 are also useful for obtaining another version of parabolic character sheaves. Lusztig's variety $Z$ is a quotient $G \times G / \Delta$, where $\Delta$ is a subgroup of $G \times G$, see below. The diagonal $G_{d}$ acts on the quotient on 
the left, so we have an action of $G_{d} \times \Delta$. Applying the results of Section 1 we connect parabolic character sheaves on $Z=G \times G / \Delta$ with certain irreducible perverse sheaves on $G=G_{d} \backslash G \times G$, transferring everything to $G$.

The group $\Delta$, introduced in 1.8, involves two parabolic subgroups of $G$ with isomorphic Levi groups. In the case that they coincide we have a parabolic subgroup $P$, with Levi group $L$ and unipotent radical $U$ and

$$
\Delta=\left\{\left(l u, l u^{\prime}\right) \in G \times G \mid l \in L, u, u^{\prime} \in U\right\} .
$$

Similar groups occur in various places in the literature (see e.g. [EL]).

In Section 2 we introduce $\Delta$-character sheaves on $G$. It uses some material from the previous paper [Sp2], which is about decompositions into "stable pieces". Section 3 deals with the connection with Lusztig's character sheaves.

Acknowledgement. Part of this work was done in February of 2008 during a stay at the Mathematical Sciences Research Institute in Berkeley. I thank MSRI for its hospitality.

\section{Preliminaries.}

1.1. $k$ is an algebraically closed field. All algebraic varieties occurring will be over $k$.

Let $X$ be an algebraic variety. We denote by $\mathcal{D}(X)$ the derived category of bounded constructible complexes of vector spaces over $E=\overline{\mathbb{Q}}_{l}$. By $\mathcal{P}(X)$ we denote the corresponding abelian category of perverse sheaves on $X$.

\subsection{Group actions.}

Let $G$ be a linear algebraic group acting on the variety $X$. We denote by $\mathcal{F}(X, G)$ the family of $G$-stable subsets of $X$. Clearly, it is stable under taking unions, intersections, complements and closures. We shall say that a bijection between two families of the type $\mathcal{F}(Z, A)$ is good if it respects unions, complements and closures. It then also respects inclusions, intersections and locally closed sets.

Let $G$ and $H$ be linear algebraic groups and let $X$ be a variety with a faithful $G \times H$-action. Assume that a quotient $H \backslash X$ exists and denote by $\pi$ the 
projection $X \rightarrow H \backslash X$ ( $G$ and $H$ are viewed as subgroups of $G \times H$ in the obvious manner). Then $G$ acts on $H \backslash X$.

1.3. Lemma. (i) $\pi$ induces a good bijection of $\mathcal{F}(X, G \times H)$ onto $\mathcal{F}(H \backslash X, G)$; (ii) Assume moreover that a quotient $G \backslash X$ also exists. Then there exists a good bijection

$$
\gamma=\gamma_{X, G, H}: \mathcal{F}(H \backslash X, G) \rightarrow \mathcal{F}(G \backslash X, H) .
$$

It induces a bijection of $G$-orbits in $H \backslash X$ onto $H$-orbits in $G \backslash X$.

Proof. See [Sp2] for the case that $G$ and $H$ are connected. The extension to the general case offers no difficulties.

\subsection{Equivariant perverse sheaves.}

We will need a complement to 1.3 concerning equivariant perverse sheaves. Let $G$ and $X$ be as before. If $G$ is connected the notion of a $G$-equivariant perverse sheaf on $X$ is well-known. Lusztig has defined the notion in the general case in the following manner, see [L3, 1.6].

For each component $C$ of $G$ let $m_{C}$ be the product map $C \times X \rightarrow X$. Denoting by $p_{C, C^{\prime}}$ the product map $C \times C^{\prime} \rightarrow C C^{\prime}$ we have

$$
m_{C} \circ\left(1, m_{C^{\prime}}\right)=m_{C C^{\prime}} \circ\left(p_{C, C^{\prime}}, 1\right) .
$$

Let $E_{C}$ be the constant sheaf on $C$ and denote by $\tilde{m}_{C}(K)$ the complex $E_{C} \otimes K$ on $C \times X$.

A $G$-equivariant structure on the perverse sheaf $K \in \mathcal{P}(X)$ gives for each component $C$ an isomorphism of semisimple complexes

$$
\phi_{C}: \tilde{m}_{C}(K) \rightarrow m_{C}^{*} K
$$

By (1) we have

$$
\left(\left(p_{C, C^{\prime}}^{*} \otimes 1\right) \circ m_{C C^{\prime}}^{*}\right)(K)=\left(\left(1 \otimes m_{C^{\prime}}^{*}\right) \circ m_{C}^{*}\right)(K) .
$$

We also have

$$
\left(\left(p_{C, C^{\prime}}^{*} \otimes 1\right) \circ \tilde{m}_{C C^{\prime}}\right)(K)=\left(\left(1 \otimes \tilde{m}_{C^{\prime}}\right) \circ \tilde{m}_{C}\right)(K) .
$$


The isomorphisms $\phi_{C}: \tilde{m}_{C}(K) \rightarrow m_{C}^{*}(K)$ are required to be compatible with (2) and (3), in an obvious sense.

Denote by $\mathcal{P}_{G}(X)$ the abelian category of perverse sheaves on $X$ with a $G$ equivariant structure. We call them, briefly, $G$-equivariant perverse sheaves on $X$.

1.5. Lemma. (i) Let $f: X \rightarrow Y$ be a locally trivial fiber space with group $G$ (for the étale topology). $K \in \mathcal{P}(X)$ is $G$-equivariant if and only if there exists $L \in \mathcal{P}(Y)$ with $K=f^{*} L[\operatorname{dim} G]$;

(ii) $f^{*}[\operatorname{dim} G]$ defines a bijection $\mathcal{P}(Y) \rightarrow \mathcal{P}_{G}(X)$ and an equivalence of these categories.

Proof. (ii) is a consequence of (i). (i) is due to Lusztig if $G$ is connected, see [MS1, Lemma 1.4.2].

To prove (i) in general we may assume that $X$ is a product $G \times Y, G$ acting on the left in the first factor and $f$ being the second projection. $f_{C}$ is the restriction of $f$ to $C \times Y$. Applications of (i) for the identity component $G^{\circ}$ give for each component $C$ a perverse sheaf $L_{C}$ on $Y$ such that the restriction $K$ to $C \times Y$ is $f_{C}^{*} L_{C}[\operatorname{dim} G]$. We have the $G / G^{\circ}$-equivariant complex $\oplus_{C} L_{C}$ on $G / G^{\circ} \times Y$. The proof is then reduced to the case that $G$ is finite, which we leave to the reader (cf. $[$ L3, 1.6]).

Now assume that $G, H$ and $X$ are as in 1.2 and that quotients $G \backslash X$ and $H \backslash X$ exist. $\gamma$ is as in Lemma 1.3.

1.6. Proposition. (i) There exists a bijection

$$
\Gamma=\Gamma_{X, G, H}: \mathcal{P}_{G}(H \backslash X) \rightarrow \mathcal{P}_{H}(G \backslash X)
$$

defining an equivalence of categories;

(ii) Let $O$ be a G-orbit in $X$ and let $x \in O, y \in \gamma(O)$. For $K \in \mathcal{P}_{G}(H \backslash X)$ we have

$$
\left(H^{*-\operatorname{dim} G} K\right)_{x}=\left(H^{*-\operatorname{dim} H}(\Gamma K)\right)_{y} .
$$

Proof. This is a consequence of Lemma 1.5.

\subsection{Notations.}


From now on $G$ is a connected, reductive, linear algebraic group over our algebraically closed field $k$. Fix a maximal torus $T$ of $G$. The root system of $(G, T)$ is $R$ and $R^{+} \subset R$ is a system of positive roots. Its set of simple roots is $I$. We identify it with the set of simple reflections. $l$ is the corresponding length function on $W$.

The Weyl group $N_{G}(T) / T$ is denoted by $W$. For $w \in W$ we denote by $\dot{w}$ a representative of $w \in N_{G}(T)$. If $H$ is a subgroup of $G$ containing $T$ and if $w \in W$ we write ${ }^{w} H=\dot{w} H \dot{w}^{-1}$.

For $J \subset I$ denote by $R_{J} \subset R$ the subsystem with basis $J$. Denote by $W_{J}$ its Weyl group and by $W^{J}$ the set of minimal length coset representatives of $W / W_{J}$. Then

$$
W^{J}=\left\{w \in W \mid w . J \subset R^{+}\right\} .
$$

$w_{0, J}$ denotes the longest element of $W_{J}$. Then $w_{0, I} w_{0, J}$ is the longest element of $W^{J}$. For $J, K \subset I$ put $W^{J, K}=\left(W^{J}\right)^{-1} \cap W^{K}$.

$P_{J}$ denotes the standard parabolic subgroup defined by $J$, so $P_{I}=G$ and $P_{\emptyset}$ is a Borel group. The Levi group of $P_{J}$ containing $T$ is $L_{J}$ and $U_{J}$ is the unipotent radical of $P_{J}$.

We have a bijection $W^{J, K} \rightarrow P_{J} \backslash G / P_{K}$ sending $w \in W^{J, K}$ to $P_{J} \dot{w} P_{K}$.

The unipotent radical of a parabolic subgroup $P$ is denoted by $U_{P}$ and the full radical by $R_{P}$.

If $P$ and $Q$ are parabolic subgroups we write $P \wedge Q=(P \cap Q) \cdot U_{P}$. This is a parabolic subgroup contained in $P$.

All parabolic subgroups occurring in the sequel will contain $T$.

\subsection{The group $\Delta_{G, P, Q, \sigma}$.}

Let $P$ and $Q$ be parabolic subgroups. There exist unique $J, K \subset I$ and $x \in$ $W^{J}, y \in W^{K}$ such that $P={ }^{x} P_{J}$ and $Q={ }^{y} P_{K}$. Let $B={ }^{x} P_{\emptyset}, C={ }^{y} P_{\emptyset}$. These are Borel subgroups of $P$, respectively, $Q$. The Levi group ${ }^{x} L_{J}$ of $P$ containing $T$ is denoted by $L$. Similarly, we have the Levi group $M$ of $Q$. The unipotent radicals of $P$ and $Q$ are $U$ and $V$. The projections $P \rightarrow L$ and $Q \rightarrow M$ are denoted by $\pi_{P}$ and $\pi_{Q}$.

Put $x W^{J, K} y^{-1}=W_{G}^{P, Q}$ (or simply $W^{P, Q}$ ). We have the decomposition of $G$ into 
locally closed pieces

$$
G=\coprod_{u \in W^{P, Q}} P u Q
$$

1.9. Assume given an isomorphism (of algebraic groups) $\sigma: L \rightarrow M$ which stabilizes $T$ (a subgroup of both $L$ and $M$ ).

With these notations define

$$
\Delta=\Delta_{G, P, Q, \sigma}=\left\{(p, q) \in P \times Q \mid \pi_{Q}(q)=\sigma\left(\pi_{P}(p)\right)\right\} .
$$

Then

$$
\delta=\operatorname{Int}\left(\dot{y}^{-1}\right) \circ \sigma \circ \operatorname{Int}(\dot{x})
$$

is an isomorphism $L_{J} \rightarrow L_{K}$, stabilizing $T$. It induces an isomorphism, also denoted by $\delta$, of the root system $R_{J}$ of $\left(L_{J}, T\right)$ with basis $J$, onto the similar system $R_{K}$. We say that $\sigma$ is standard if $\delta(J)=K$. It follows from the definitions that then $C=\sigma(B)$.

1.10. $\Delta$ is a subgroup of $G \times G$. It acts on $G$ by $(p, q) \cdot x=p x q^{-1}$. The pieces of the decomposition (4) are stable under the $\Delta$-action.

Notice that $2 \operatorname{dim} U=\operatorname{dim} G-\operatorname{dim} L=\operatorname{dim} G-\operatorname{dim} M=2 \operatorname{dim} V$. It follows that

$$
\operatorname{dim} \Delta=\operatorname{dim} L+\operatorname{dim} U+\operatorname{dim} V=\operatorname{dim} L+2 \operatorname{dim} U=\operatorname{dim} G .
$$

Also notice that $(p, q) \mapsto(q, p)$ induces an isomorphism

$$
\Delta_{G, P, Q, \sigma} \rightarrow \Delta_{G, Q, P, \sigma^{-1}}
$$

and that for $w \in W$

$$
\Delta_{G,{ }^{w} P,{ }^{w} Q, \operatorname{Int}(w) \circ \sigma}={ }^{w} \Delta_{G, P, Q, \sigma} .
$$

Groups like $\Delta$ occur in the work of others, for example in [EL].

1.11. Example. If $P=Q=G$ then $x=y=1$ and $B=C=P_{\emptyset}$. The automorphism $\sigma$ of $G$ is standard if and only if it stabilizes both $T$ and $B$. Now $\Delta \simeq G$ and the action on $G$ becomes $\sigma$-twisted conjugacy : $(g, x) \mapsto g x(\sigma(g))^{-1}$.

\section{2. $\Delta$-character sheaves.}


2.1. Let $\Delta=\Delta_{G, P, Q, \sigma}$. as before. Now $B$ is an arbitrary Borel group of $P$ containing $T$. We have $B=(B \cap L) \cdot U$ and $B \cap L$ is a Borel group of $L$. Also $C=\sigma(B \cap L) . V$ is a Borel group of $Q$. Put $\Delta^{\prime}=\Delta_{G, B, C,\left.\sigma\right|_{T}}$ and

$$
B_{1}=\left\{(u b, \sigma(b) v) \mid u \in U, v \in V, b \in B_{L}\right\} .
$$

This is a Borel subgroup of $\Delta$, contained in $\Delta^{\prime}$.

By the Bruhat decomposition in $G$

$$
G=\coprod_{w \in W} B w C
$$

All $B w C$ are stable under the action on $G$ of $\Delta^{\prime} \subset \Delta$. Write $d(w)=\operatorname{dim} B w C$.

2.2. Fix $w \in W$. We have a morphism $\rho: B w C \rightarrow T$ sending $u t \dot{w} v \in B w C$ to $t$ $\left(u \in U_{B}, v \in U_{C}, t \in T\right)$. $\rho$ depends on the choice of $\dot{w}$.

If $z=(u t, \sigma(t) v) \in B_{1}(u \in U, v \in V, t \in T)$ and $y \in B w C$ then

$$
\rho(z . y)=t \rho(y)(w \sigma(t))^{-1}
$$

As in [MS2, 2.1.1] let $\hat{X}(T)=X(T) \otimes \mathbb{Z}_{(p)} / \mathbb{Z}$ be the group of tame (or Kummer) local systems on $T$, where $X(T)$ is the character group of $T$ and $p=\operatorname{char}(k)$.

For $\xi \in \hat{X}(T)$ put $\mathcal{L}_{\xi, \dot{w}}=\rho^{*} \xi$. If $w \sigma(\xi)=\xi$ then $\mathcal{L}_{\xi, \dot{w}}$ is $\Delta^{\prime}$-equivariant. Let $A_{\xi, \dot{w}}$ be its perverse extension. It is a $\Delta^{\prime}$-equivariant perverse sheaf on $G$ whose restriction to $B w C$ is $\mathcal{L}_{\xi, \dot{w}}[\operatorname{dim} B w C]$ and whose support is $\overline{B w C}$.

$E[\operatorname{dim} G] \otimes A_{\xi, \dot{w}}$ is a perverse sheaf on $\Delta \times G$, which up to a shift is the pull-back of a perverse sheaf $\tilde{A}$ on the quotient $\Delta \times_{B_{1}} G$ ( $B_{1}$ acting on the right on $\Delta$ and via the $\Delta$-action of $G$ on $B w C)$. Denote by

$$
a: \Delta \times{ }_{B_{1}} G \rightarrow G
$$

the morphism induced by the action map. It is a proper morphism.

By the decomposition theorem, $C_{\xi, \dot{w}}^{B}=C_{\xi, \dot{w}}=(a) ! \tilde{A}$ is a semi-simple complex. A $\Delta$-character sheaf on $G$ is an irreducible perverse sheaf $A$ such that some shift $A[i]$ is a direct summand of a $C_{\xi, \dot{w}}$. Write $d(A)$ for the minimal value $d(w)$ for which this happens.

The isomorphism class of $C_{\xi, \dot{w}}$ does not depend on the choice of $\dot{w}$; we write $C_{\xi, w}$ if only its isomorphsm class matters. 
Let $B^{\prime} \supset T$ be another Borel group of $P$. Then $B^{\prime}={ }^{x} B$ with $x \in W_{L}$, the Weyl group of $(L, T)$. Put $C^{\prime}=\sigma\left(B^{\prime} \cap L\right) V$, a Borel group of $Q$. We can perform the construction of $\Delta$-character sheaves with $B^{\prime}, C^{\prime}$ instead of $B, C$.

2.3. Lemma. $C_{x . \xi, x w(\sigma x)^{-1}}^{B^{\prime}}$ is isomorphic to $C_{\xi, w}^{B}$.

We skip the easy proof.

It follows that the notion of $\Delta$-character sheaf is independent of the choice of $B$.

2.4. Example. In the case $P=Q=G$ we call the $\Delta$-character sheaves $\sigma$ twisted character sheaves on $G$. They are related to Lusztig's character sheaves on non-connected groups. For $\sigma=$ id one obtains the usual character sheaves.

Denote by $j=j_{w}$ the inclusion $B w C \rightarrow G$. The sheaf $E \otimes j_{!}\left(\mathcal{L}_{\xi, \dot{w}}\right)$ is the pull-back of a sheaf $\tilde{\mathcal{L}}$ on $\Delta \times_{B_{1}} G$. Put $K_{\xi, \dot{w}}=a_{!} \tilde{\mathcal{L}}$.

$T_{1}=\left\{(t, \sigma(t) \mid t \in T\}\right.$ is a maximal torus of $B_{1}$, isomorphic to $T$ via the first projection. We introduce the quotient $\Delta \times_{T} B w, T$ acting on $\Delta$ on the right via its subgroup $T_{1}$ and on $B w C$ by twisted conjugacy $\left(t . x=t x(\sigma t)^{-1}\right)$. We have a morphism $q: \Delta \times_{T} B w \rightarrow \Delta \times_{B_{1}} B w C$.

2.5. Lemma. (i) $q$ is surjective;

(ii) Its fibers are isomorphic to an affine space $\mathbb{A}^{m}$ and $q^{*} \tilde{\mathcal{L}}$ is constant along the fibers.

Proof. (i) is clear. (ii) follows from the fact that the fibers are isomorphic to a subgroup $U^{\prime}$ of $U$, namely the set of unipotent $u \in B_{L}$ with $\sigma(u) v \in{ }^{w^{-1}} B \cap C$ for some $v \in V$.

Put $a^{\prime}=a \circ q, \breve{\mathcal{L}}=q^{*} \tilde{\mathcal{L}}, L_{\xi, \dot{w}}=a_{!}^{\prime} \breve{\mathcal{L}}$.

If $A$ is an irreducible perverse sheaf on the variety $X$ and $K \in \mathcal{D}(X)$ we write $A \dashv K$ if $A$ is a composition factor of some ${ }^{p} H^{i}(A)$.

2.6. Lemma. (i) An irreducible perverse sheaf $A$ on $G$ is a $\Delta$-character sheaf if and only if $A \dashv K_{\xi, \dot{w}}$ for some $\xi, w$. In that case $d(A) \leq d(w)$;

(ii) Same as (i) with $K_{\xi, \dot{w}}$ replaced by $L_{\xi, \dot{w}}$.

Proof. The proof of the first part of (i) is similar to the proof of $[\mathrm{L} 1,12.7]$. The 
second part also follows.

To prove (ii) use that as a consequence of Lemma 2.5 (ii)

$$
q ! \breve{\mathcal{L}}=q ! q^{*}(\tilde{\mathcal{L}})=\tilde{\mathcal{L}}[-m]
$$

and $L_{\xi, \dot{w}}=K_{\xi, \dot{w}}[-m]$.

Let $A$ be a $\Delta$-character sheaf.

2.7. Lemma. Assume $w$ and $\xi$ are such that $A \dashv C_{\xi, \dot{w}}$, with $d(A)=d(w)$. Let $u \in W^{P, Q}$ be such that $P u Q=P w Q$.

(i) $P u Q \cap \operatorname{Supp}(A)$ is open in $\operatorname{Supp}(A)$;

(ii) $u$ is uniquely determined by $A$;

(iii) $A \dashv L_{\xi, \dot{w}}$.

Proof. The notations are as in 2.2. Put $K=C_{\xi, \dot{w}}$. There is $h$ such that $A[h]$ is a direct summand of $K$.

We have $\operatorname{Supp}(A) \subset \overline{P u Q}$. If the lemma were false, $\operatorname{Supp}(A)$ would lie in a set $\overline{P u^{\prime} Q} \subsetneq \overline{P u Q}$. Let $i$ be the inclusion $\overline{P u^{\prime} Q} \rightarrow X$. Then $A=i_{*} B$, where $B \in \mathcal{P}\left(\overline{P u^{\prime} Q}\right)$.

Put $S=a^{-1}\left(\overline{P u^{\prime} Q}\right)$, a closed subset of $\Delta \times_{B_{1}} G$. Let $\tilde{A}^{\prime}$ be the restriction of $\tilde{A}$ to $S$ and let $a^{\prime}$ be the restriction of $a$ to $S$. By base change, $\left(a^{\prime}\right) ! \tilde{A}^{\prime}$ has a direct summand $B[h]$, hence $B \dashv\left(a^{\prime}\right) ! \tilde{A}^{\prime}$. But $S$ is a union of sets $\Delta \times_{B_{1}} B x C$ with $d(x)<d(w)$. From this we conclude that $A \dashv K_{\eta, \dot{x}}$, for some $x$ with $d(x)<d(w)$. By Lemma 2.6 (i) this contradicts the assumption $d(A)=d(w)$.

Then (ii) follows from the existence of the decomposition (4). The proof of (i) also gives that $A \dashv K_{\xi, \dot{w}}$, which implies (iii) since $L_{\xi, \dot{w}}$ is a shift of $K_{\xi, \dot{w}}$ (see the proof of Lemma 2.6(ii))

2.8. We introduce some notations used in a further analysis of the complexes $L_{\xi, \dot{w}}$, see [Sp2, sect. 2].

Let $u \in W^{P, Q}$. Put $Z={ }^{-1} P \cap Q$. We have a decomposition with uniqueness

$$
Z=\left({ }^{u^{-1}} L \cap M\right) \cdot\left({ }^{u^{-1}} L \cap V\right) \cdot\left({ }^{u^{-1}} U \cap M\right) \cdot\left({ }^{u^{-1}} U \cap V\right) .
$$

$Q \wedge{ }^{u^{-1} P}=Z . V$ is a parabolic subgroup contained in $Q$. Its image in $M$ is a parabolic subgroup of $M$, which we write as $\sigma\left(P_{1}\right)$, where $P_{1}$ is a parabolic subgroup of $L$. 
$L_{1}=\sigma^{-1}\left(u^{-1} L \cap M\right)$ is the Levi group of $P_{1}$ containing $T$ and $U_{1}=\sigma^{-1}\left(u^{-1} U \cap M\right)$ is its unipotent radical.

Likewise, $P \wedge{ }^{u} Q$ is a parabolic subgroup contained in $P$. Its image $Q_{1}$ in $L$ is a parabolic with Levi group $M_{1}=L \cap{ }^{u} M$ and unipotent radical $L \cap{ }^{u} V$.

$\operatorname{Int}(\dot{u}) \circ \sigma$ induces an isomorphism $\sigma_{1}$ of $L_{1}$ onto $M_{1}$, It is determined by $\sigma$ and $u$ up to an inner automorphism $\operatorname{Int}(t)(t \in T)$.

By [loc. cit., Lemma 2.4] $\sigma_{1}$ is standard if $\sigma$ is.

Put $\Delta_{1}=\Delta_{L, P_{1}, Q_{1}, \sigma_{1}}$.

Then

$$
\zeta=\left(\pi_{P_{1}} \circ \sigma^{-1} \circ \pi_{Q}, \pi_{Q_{1}} \circ \pi_{P} \circ \operatorname{Int}(\dot{u})\right)
$$

defines a surjective homomorphism $Z \rightarrow \Delta_{1}$, whose kernel is a connected unipotent group (cf. [Sp2, 2.5 (iii)]). For $t \in T$ we have

$$
\zeta(z t)=\zeta(z)\left(t, \sigma_{1}(t)\right)
$$

(recall that $\Delta_{1}$ is a subgroup of $P_{1} \times Q_{1}$ ).

2.9. Let $L_{\xi, \dot{w}}$ be as before. There is a unique $u \in W^{P, Q}$ with $P w Q=P u Q$. Denote by $j^{\prime}: P u Q \rightarrow G$ the inclusion. We have a morphism

$$
\alpha: \Delta \times_{T} B w \rightarrow P u Q
$$

such that $a^{\prime}=j^{\prime} \circ \alpha$. Then

$$
L_{\xi, \dot{w}}=j_{!}^{\prime}(\alpha ! \breve{\mathcal{L}})
$$

Denote by $W_{L}$ the Weyl group of $(L, T)$. There exist $w_{1}, x \in W_{L}$ with $w=$ $w_{1} u(\sigma x)$. In fact, by a result of Howlett (see [C, Prop. 2.7.5]) we may assume that $w_{1} \in W_{L}^{B_{1}, Q_{1}}$, in which case $w_{1} u \in W_{G}^{B, Q}$ (moreover the decomposition is unique). It follows from Lemma 2.3 that after replacing $B$ by ${ }^{x} B$ we are in the situation that $w \in W_{G}^{B, Q}$ (i.e. $x=1$ ). This we assume from now on.

2.10. $Z$ acts on $P \times Q$ by $z \cdot(p, q)=\left(p \dot{u} z^{-1} \dot{u}^{-1}, z q\right)$. Let $\beta: P \times Q \rightarrow P u Q$ be the map sending $(p, q)$ to $p \dot{u} q$. This is a quotient map for the $Z$-action on $P \times Q$ 
(see $[\mathrm{Sp} 2,2.5])$.

Consider $\Delta \times B \times Z$. The maximal torus $T$ operates on it by

$$
t .((p, q), b, z)=\left(\left(p t^{-1}, q(\sigma(t))^{-1}\right), t b(w \sigma(t))^{-1}, z \sigma(t)^{-1}\right) .
$$

The quotient variety relative to this action is denoted by $\mathcal{Z}$. The morphism $\gamma^{\prime}: \Delta \times B \times Z \rightarrow P \times Q$ with $\left.\gamma^{\prime}((p, q), b, z)\right)=\left(p b \dot{w}_{1} \dot{u} z^{-1} \dot{u}^{-1}, z q^{-1}\right)$ is constant on $T$-orbits and defines a morphism $\gamma: \mathcal{Z} \rightarrow P \times Q$. Let $\delta: \mathcal{Z} \rightarrow \Delta \times_{T} B w$ be the morphism sending the image of $((p, q), b, z)$ to the image of $((p, q), b w)$. Let $\xi^{\prime}$ be the pull-back to $B$ of the local system $\xi$ on $T$. Recall that $w \sigma(\xi)=\xi$. Under the assumption $x=1$ of 2.9 this implies that also $\left(w_{1} \sigma_{1}\right) \xi=\xi$.

2.11. We have a morphism

$$
\alpha_{1}: \Delta_{1} \times_{T} B_{1} w_{1} \rightarrow L
$$

similar to the morphism $j^{\prime} \circ \alpha$ of the previous setting. Let $\breve{\mathcal{L}}_{1}$ be the local system on $\Delta_{1} \times_{T} B_{1} w_{1}$ corresponding to $\breve{\mathcal{L}}$. Let

$$
\theta: Z \times_{T} B_{1} w_{1} \rightarrow \Delta_{1} \times_{T} B_{1} w_{1}
$$

be the morphism induced by $\left(\zeta\right.$,id) and put $\bar{\alpha}_{1}=\alpha_{1} \theta$.

$\delta_{1}: \mathcal{Z} \rightarrow Z \times_{T} B_{1} w_{1}$ maps the image of $((p, q), b, z)$ to the image of $\left(z, \pi_{P}(b) w_{1}\right)$. Define $\epsilon: P \times Q \rightarrow L$ by $\epsilon(p, q)=\sigma^{-1}\left(\pi_{Q}(q)\right) \pi_{P}(p)$. By [Sp2, Lemma 2.5 (ii)] it is a quotient map for the $\Delta$-action on $G$. The group $Z$ acts on it via its quotient $\Delta_{1}$.

Consider the diagram

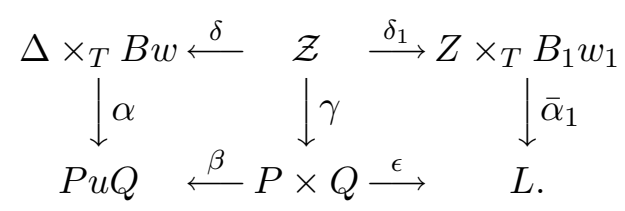

2.12. Lemma. (i) The two squares of (9) are pull-back diagrams;

(ii) $E \otimes \xi^{\prime} \otimes E$ is the pull-back to $\Delta \times B \times Z$ of both $\delta^{*} \breve{\mathcal{L}}$ and $\delta_{1}^{*}\left(\theta^{*} \breve{\mathcal{L}}_{1}\right)$;

(iii) $\beta^{*}\left(\alpha_{!} \breve{\mathcal{L}}\right)=\gamma_{!}\left(\delta^{*} \breve{\mathcal{L}}\right)=\epsilon^{*}\left(\left(\bar{\alpha}_{1}\right) !\left(\theta^{*} \breve{\mathcal{L}}_{1}\right)\right)$.

Proof. The proofs of (i) and (ii) are straightforward. (iii) follows by base change. 
2.13. Let $A$ be a $\Delta$-character sheaf on $G$. Assume that $A \dashv L_{\xi, \dot{w}}$. Let $u \in W^{P, Q}$ be such that $P w Q=P u Q$ and that $P u Q \cap \operatorname{Supp}(A)$ is open in $\operatorname{Supp}(A)$ (see Lemma 2.7). We assume that $w=w_{1} u$ with $w_{1} \in W_{L}$ (see 2.9).

$j^{\prime}$ being, as before, the inclusion map $P u Q \rightarrow G,\left(j^{\prime}\right)^{*} A$ is an irreducible perverse sheaf on $P u Q$ which is $\Delta$-equivariant. It determines $A$ uniquely.

With the notations of Prop. 1.6 there corresponds to $\left(j^{\prime}\right)^{*} A$ an irreducible perverse sheaf $A_{1}=\Gamma_{P \times Q, \Delta, Z}\left(j^{*} A\right)$ on $L$ which is $Z$-equivariant, i.e. $\Delta_{1}$-equivariant because $Z$ acts on $L$ via its quotient $\Delta_{1}$.

2.14. Lemma. $A_{1}$ is a $\Delta_{1}$-character sheaf on $L$.

Proof. Notations are as before. Assume that $\left(j^{\prime}\right)^{*} A$ is a composition factor of ${ }^{p} H^{i}(\alpha ! \breve{\mathcal{L}})$.

$\beta^{*}\left(j^{\prime}\right)^{*} A[\operatorname{dim} Z]$ is an irreducible perverse sheaf on $P \times Q$ which is $\Delta \times Z$ equivariant. Since $\beta^{*}[\operatorname{dim} Z]$ is a $t$-exact functor, there is an $i$ such that this perverse sheaf is a composition factor of

$$
{ }^{p} H^{i+\operatorname{dim} Z}\left(\beta^{*} \alpha ! \breve{\mathcal{L}}\right)={ }^{p} H^{i+\operatorname{dim} Z}\left(\gamma ! \delta^{*} \breve{\mathcal{L}}\right),
$$

by Lemma 2.12 (iii).

Similarly, the composition factors of ${ }^{p} H^{i+\operatorname{dim} G}\left(\gamma_{!} \delta_{1}^{*} \breve{\mathcal{L}}\right)$ correspond to composition factors of ${ }^{p} H^{i}\left(\left(\bar{\alpha}_{1}\right) !\left(\theta^{*} \breve{\mathcal{L}}_{1}\right)\right)$.

Now we see from (8) that $\theta$ is a fibration whose fibers are affine spaces (isomorphic to $\operatorname{Ker}(\zeta))$. Hence

$$
\left(\bar{\alpha}_{1}\right) !\left(\theta^{*} \breve{\mathcal{L}}_{1}\right)=\left(\alpha_{1}\right) !\left(\theta_{!} \theta^{*} \mathcal{L}_{1}\right)=\left(\alpha_{1}\right) ! \mathcal{L}_{1}[-c]
$$

where $c$ is the fiber dimension.

We conclude that the irreducible constituents of the ${ }^{p} H^{j}\left(\gamma_{!} \delta^{*} \breve{\mathcal{L}}\right)$ are of the form $\epsilon^{*} A^{\prime}$, where $A^{\prime}$ is a $\Delta_{1}$-character sheaf. Since $\beta^{*}\left(j^{\prime}\right)^{*} A[\operatorname{dim} Z]$ is such a constituent, the Lemma follows from the definition of the bijection $\Gamma_{P \times Q, \Delta, Z}$.

\subsection{The $\Delta$-stable pieces.}

For $w \in W^{B, Q}$ put $G_{w}=\Delta .(B w)$. The following is proved in [Sp2, Theorem 2.6]:

(a) $G$ is the disjoint union of the $G_{w}\left(w \in W^{B, Q}\right)$,

(b) For each $w \in W^{B, Q}$ there is a connected reductive subgroup $H_{G, w}=H_{w} \supset T$ of $G$ together with an automorphism $\tau_{G, w}=\tau_{w}$ of $H_{w}$ such that there is a good 
bijection $\gamma_{w}$ of $\mathcal{F}\left(G_{w}, \Delta\right)$ onto $\mathcal{F}_{\tau_{w}}\left(H_{w}, H_{w}\right)$ (the suffix $\tau_{w}$ denoting twisted conjugacy action). Moreover $\tau_{w}$ fixes $T$ and a Borel group containing $T$.

(a) and (b) are counterparts to the results about "stable pieces" of Lusztig and He (see [H1, H2]). The proof of (a) and (b) uses the constructions of 2.8.

We next give complements about perverse sheaves. Denote by $\operatorname{Irr}_{\Delta} G$ the set of isomorphism classes of irreducible $\Delta$-equivariant perverse sheaves on $G$ and by $\operatorname{Char}_{\Delta}(G)$ the set of isomorphism classes of $\Delta$-character sheaves on $G$. By $\operatorname{Irr}_{H_{w}, \tau_{w}}\left(H_{w}\right)$ and $\operatorname{Char}_{\tau_{w}}\left(H_{w}\right)$ we denote irreducible perverse sheaves on $H_{w}$ equivariant under $\tau_{w}$-twisted conjugation, respectively, the corresponding twisted character sheaves.

2.16. Theorem. (i) There is a bijection

$$
\Gamma_{\Delta}: \operatorname{Irr}_{\Delta}(G) \rightarrow \coprod_{w \in W^{B, Q}} \operatorname{Irr}_{H_{w}, \tau_{w}}\left(H_{w}\right)
$$

(ii) It induces a bijection

$$
\operatorname{Char}_{\Delta}(G) \rightarrow \coprod_{w \in W^{B, Q}} \operatorname{Char}_{\tau_{w}}\left(H_{w}\right)
$$

Proof. Notations are as before. Let $A$ be an irreducible perverse sheaf on $X$. There is a unique $u \in W^{P, Q}$ such that $P u Q \cap \operatorname{Supp}(A)$ is open in $\operatorname{Supp}(A)$. The restriction of $A$ to $P u Q$ is an irreducible $\Delta$-equivariant perverse sheaf on $P u Q=Z \backslash P \times Q$. Applying Prop. 1.6 we obtain a perverse sheaf $A_{1}$ on $\Delta \backslash P \times Q=L$, which is $Z$-equivariant i.e. $\Delta_{1}$-equivariant since $Z$ acts via its quotient $\Delta_{1}$ (see 2.11). We may assume that $P \neq G$. Then $\operatorname{dim} L<\operatorname{dim} G$. As in the proof of [Sp2, Th. 2.6] we use induction.

We may assume that the theorem holds for $L$ and $\Delta_{1}$. So we have attached to $A_{1}$ an element $w_{1} \in W_{L}^{B \cap L, Q_{1}}$ and $A^{\prime} \in \operatorname{Irr}_{H_{L, w_{1}}, \tau_{L, w_{1}}}\left(H_{L, w_{1}}\right)$. We have $G_{w}=\gamma_{P \times Q, Z, \Delta} L_{w_{1}}$ and $H_{w}=H_{L, w_{1}}, \tau_{w}=\tau_{L, w_{1}}$ (see the proof of [loc. cit.]). Moreover $\Gamma_{P \times Q, Z, \Delta} A^{\prime}$ is the restriction of $A$ to $P u Q$. These facts imply (i). Then (ii) is a consequence of Lemma 2.14 .

Assume that $P \neq G$. Let $A \in \operatorname{Irr}_{\Delta}(G)$ and assume that $A^{\prime}=\Gamma_{\Delta} A \in \operatorname{Irr}_{H_{w}, \tau_{w}}\left(H_{w}\right)$. With the notations of 1.7 and 1.8 we have $w=x \bar{w} y^{-1}$ where $\bar{w} \in W^{K}$. 
2.17. Corollary. Let $O$ be a $\Delta$-orbit in $G_{w}$ and let $p \in O, q \in \gamma_{w}(O)$. Then

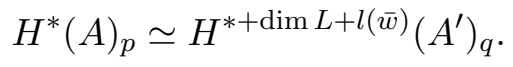

Proof. By Prop. 2.6 (ii)

$$
H^{*}(A)_{p}=H^{*+\operatorname{dim} \Delta-\operatorname{dim} Z}\left(A^{\prime}\right)_{p_{1}},
$$

where $p_{1}$ lies in the $\Delta_{1}$-orbit corresponding to $O$. By induction we have equalities

$$
H^{*}\left(A_{1}\right)_{p_{1}}=H^{*+l\left(w_{1}\right)}\left(A_{1}\right)_{q} .
$$

Now by $[\mathrm{Sp} 2,(3)]$

$$
\operatorname{dim} \Delta-\operatorname{dim} Z=\operatorname{dim} G-\operatorname{dim} Z=\operatorname{dim} L-\operatorname{dim} L_{1}+l(b),
$$

with $b \in W^{J, K}$ such that $l(\bar{w})=l(b)+l\left(\bar{w}_{1}\right)$ (see the proof of [loc. cit., 2.12]). The corollary follows by induction.

2.18. Remark. The induction used in the proofs of Theorem 2.16 and of [Sp2, Theorem 2.6] could be made more explicit, leading to an approach that is in the spirit of [L2]. We sketch how to do this.

Put $L_{0}=M_{0}=G, P_{0}=P, Q_{0}=Q, \sigma_{1}=\sigma$. Iteration of the construction of 2.8 leads to sequences

$$
\mathbf{t}=\left(L_{i}, P_{i}, Q_{i}, \sigma_{i}, u_{i+1}\right)_{i \geq 0},
$$

with the following data:

(a) $L_{i}$ is a connected reductive subgroup of $G$ containing $T$ and $P_{i}, Q_{i}$ are parabolic subgroups of $L_{i}$ containing $T$. Moreover, $L_{i+1}$ and $M_{i+1}$ are the Levi subgroups containing $T$ of $P_{i}$, respectively, $Q_{i}$ and $\sigma_{i+1}$ is an isomorphism $L_{i+1} \rightarrow M_{i+1}$. Put $\Delta_{i}=\Delta_{L_{i}}\left(P_{i}, Q_{i}, \sigma_{i+1}\right)$.

(b) $u_{i} \in W_{L_{i}}^{P_{i}, Q_{i}}$. Put $Z_{i}=u_{i}^{-1} P_{i} \cap Q_{i}$.

(c) $\sigma_{i+1} P_{i+1}$ is the image in $M_{i+1}$ of $Z_{i}, Q_{i+1}$ is the image in $L_{i+1}$ of ${ }^{u_{i}} Z_{i}$ and $\sigma_{i+1}$ is induced by $\operatorname{Int}\left(\dot{u}_{i}\right) \circ \sigma_{i}$.

(d) $Z_{i} \times \Delta_{i}$ acts on $P_{i} \times Q_{i}$ and $Z_{i} \backslash\left(P_{i} \times Q_{i}\right)=P_{i} u_{i+1} Q_{i} \subset L_{i}, \Delta_{i} \backslash\left(P_{i} \times Q_{i}\right)=L_{i+1}$. There is $n \geq 0$ such that $L_{n}=L_{n+1}$, in which case $L_{n}=P_{n}=Q_{n}$. Then $\sigma_{n}$ is an automorphism of $L_{n}$ which fixes $T$ and a Borel group of $L_{n}$ containing $T$, by 
the results of [Sp2]. Notice that $\mathcal{F}\left(L_{n}, \Delta_{n}\right)$ is the family of subsets of $L_{n}$ stable under $\sigma_{n}$-twisted conjugacy.

By Lemma 1.3 (ii) we have a good bijection

$$
\gamma_{i}=\gamma_{P_{i} \times Q_{i}, Z_{i}, \Delta_{i}}: \mathcal{F}\left(L_{i+1}, \Delta_{i+1}\right) \rightarrow \mathcal{F}\left(P_{i} u_{i+1} Q_{i}, \Delta_{i}\right) .
$$

Put

$$
\gamma_{\mathbf{t}}=\gamma_{0} \circ \gamma_{1} \circ \ldots \circ \gamma_{n-1}
$$

and let $G_{\mathbf{t}}=\gamma_{\mathbf{t}}\left(L_{n}\right)$. This is a $\Delta$-stable subset of $G$, in fact one of the $G_{w}$.

\section{Connection with Lusztig's parabolic character sheaves.}

3.1. We maintain the notations of the preceding section.

In [L2, sect. 9] Lusztig introduces a variety denoted by $Z_{J, y, \delta}$, where

(a) $J \subset I$,

(b) $\delta$ is an isomorphism of the Coxeter group $(W, I)$ (so $\delta I=I)$,

(c) $y \in W^{K, \delta J}$ and $y . \delta J=K$.

Let $\sigma$ be an isomorphism $L_{J} \rightarrow y^{-1} L_{K}$ stabilizing $T$ and inducing the bijection $\delta: J \rightarrow y^{-1} . K$ of simple roots.

$G \times G$ operates transitively on $Z_{J, y, \delta}$.

3.2. Lemma. There is $z \in Z_{J, y, \delta}$ whose isotropy group in $G \times G$ is $\Delta_{G, P_{J}, y^{-1} P_{K}, \sigma}$. Proof. With the notations of [loc. cit.] we may take

$$
z=\left(P_{J}, y^{-1} P_{K}, U_{Q} \dot{y}^{-1} U_{P}\right) .
$$

We consider the slightly more general varieties

$$
Z=Z_{G, P, Q, \sigma}=\Delta_{G, P, Q, \sigma} \backslash G \times G,
$$

$\Delta$ acting on $G \times G$ on the right in the obvious way (no confusion will arise with the group $Z$ of 2.8 ).

3.3. Example. Assume $G$ to be adjoint. Let $J \subset I$ and let $P_{J}^{-}$be the opposite of the standard parabolic subgroup $P_{J}$. Then $P_{J}^{-}={ }^{x} P_{\iota J}$, where $x=w_{0, I} w_{0, J}$ 
and $\iota$ is the opposition involution of $I$, induced by $-w_{0, I}$. Let $\sigma: L_{\iota J} \rightarrow L_{J}$ be the isomorphism of Levi groups induced by $\operatorname{Int}(\dot{x})$.

$C_{J}=(x, \sigma x)\left(x \in Z\left(L_{\iota J}\right)\right)$ is a torus in $G \times G$ acting freely on $Z_{G, P_{J}^{-}, P_{J}, \sigma}$. Let $X_{J}$ be the quotient variety. (Notice that because $G$ is adjoint the center of a Levi group is connected.) Then $X_{J}$ is isomorphic to a $G \times G$-orbit in the wonderful compactification of $G$.

Let $\tau$ be an automorphism of $G$. We assume that it stabilizes $T$ and our standard Borel group $P_{\emptyset}$. Put $G_{d, \tau}=(g, \tau g)(g \in G)$ (the $\tau$-twisted diagonal). $G_{d}$ is the diagonal subgroup of $G \times G$.

It is clear that $d_{\tau}:(x, y) \mapsto x^{-1}\left(\tau^{-1} y\right)$ is a quotient map for the left action of $G_{d, \tau}$. So the quotient is $G$. Put $\Delta_{\tau}=\Delta_{G, P, \tau Q, \tau \circ \sigma}, V_{\tau}=\Delta_{\tau} \backslash G \times G$. Then $G_{d, \tau}$ acts on $V_{\tau}$.

3.4. Lemma. $\left(1, \tau^{-1}\right)$ induces a morphism $V_{\tau} \rightarrow V$ intertwining the $G_{d, \tau^{-}}$ and $G_{d^{-}}$actions.

Proof. Easy.

The group $G_{d, \tau} \times \Delta_{\tau}$ acts on $G \times G$. We apply the results of section 1 for this case.

3.5. Lemma. (i) $\gamma_{G \times G, G_{d, \tau}, \Delta_{\tau}}$ defines a good bijection

$$
\mathcal{F}\left(G, \Delta_{\tau}\right) \rightarrow \mathcal{F}\left(V_{\tau}, G_{d, \tau}\right) ;
$$

(ii) $\Gamma_{G \times G, G_{d, \tau}, \Delta_{\tau}}$ defines a bijection $\mathcal{P}_{\Delta_{\tau}}(G) \rightarrow \mathcal{P}_{G_{d, \tau}}\left(V_{\tau}\right)$.

Proof. Apply Lemma 1.3 and Prop. 1.6 (i).

3.6. We shall identify the perverse sheaves on $V_{\tau}$ corresponding to the $\Delta_{\tau^{-}}$ character sheaves on $G$. It follows from Lemma 3.4 that it suffices to deal with the case $\tau=1$. Put $d=d_{1}$ and let $\phi: G \times G \rightarrow Z$ be the quotient map for the $\Delta$-action.

We use the notations of 2.1 and 2.2. The group $B_{1}$ acts on $G \times \Delta \times G$ by

$$
(x, y) \cdot(g,(p, q), h)=\left(g x^{-1},\left(p x^{-1}, q y^{-1}\right), x h y^{-1}\right),
$$

where $(x, y) \in B_{1},(p, q) \in \Delta, g, h \in G$. Let $\mathcal{U}$ be the quotient for this action. Projection on the last two factors defines a map $b: \mathcal{U} \rightarrow \Delta \times_{B_{1}} G$. We denote by 
$c$ the map $\mathcal{U} \rightarrow G \times G$ which sends the image of $(g,(p, q), h)$ to $\left(g p^{-1}, g h q^{-1}\right)$. Let $Z_{0}$ be the image in $Z$ of $\{1\} \times G$. The obvious map $p: G \rightarrow Z_{0}$ identifies $Z_{0}$ with $G / V$ and $Z_{0}$ is $B \times B$-stable. If $A \in \mathcal{P}(G)$ is equivariant for the right $V$-action there is $A_{0} \in \mathcal{P}\left(Z_{0}\right)$ with $A=p^{*} A_{0}[\operatorname{dim} V]$.

$f: \mathcal{U} \rightarrow G \times{ }_{B} Z_{0}$ maps the image of $(g,(p . q), h)$ to the image of $(g,(1, h))$. The $B$ actions on $G$ and $Z_{0}$ are, respectively, right action and the action via the diagonal $B_{d} \subset G \times G$. If $K$ is a $B \times B$-equivariant perverse sheaf on $Z_{0}$ we denote by $\breve{K}$ the perverse sheaf on $G \times{ }_{B} Z_{0}$ whose pull-back to $G \times Z_{0}$ is $E \otimes A_{1}[\operatorname{dim} G-\operatorname{dim} B]$. $q: G \times{ }_{B} Z_{0} \rightarrow Z$ sends the image of $(g, h)$ to $\phi(g, g h)$.

Consider the diagram

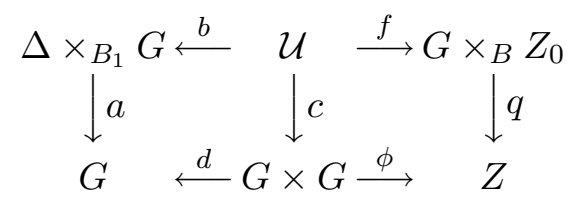

$\tilde{A}$ and $A_{\xi, \dot{w}}$ are as in 2.2 .

3.7. Lemma. (i) The two squares of (10) are pull-back diagrams;

(ii) Let $K \in \mathcal{P}(\mathcal{U})$ be the perverse sheaf on $\mathcal{U}$ whose pull-back to $G \times \Delta \times G$ is $E \otimes E \otimes \tilde{A}[\operatorname{dim} G]$. Then $K=b^{*} \tilde{A}[\operatorname{dim} G]=f^{*} \breve{A}_{0}[\operatorname{dim} G] ;$

(iii) $c_{!} K=d^{*} a_{!} \tilde{A}=\phi^{*} q ! \breve{A}_{1}$.

Proof. Similar to the proof of Lemma 2.12.

We define a parabolic character sheaf on $Z$ to be an irreducible constituent of a semisimple sheaf of the form $q_{!}\left(\breve{A}_{\xi, \dot{w}}\right)_{0}$.

3.8. Theorem. $A \in \mathcal{P}(G)$ is a $\Delta$-character sheaf if and only if $\Gamma_{G \times G, G_{d}, \Delta} A$ is a parabolic character sheaf.

Proof. This uses Lemma 3.7 in much the same way as Lemma 2.12 is used in the proof of Lemma 2.14. Details may be left to the reader.

\section{Another version of the parabolic character sheaves.}

4.1. We maintain the notations of the preceding section. The perverse sheaf $A_{0}$ on $Z_{0}$ can be described in another way. Let $O=(B \times B)$. $\phi(1, w)=p(B w C)$. The local system $\mathcal{L}=\mathcal{L}_{\xi, \dot{w}}$ on $B w C$ is the pull-back of a local system $\mathcal{L}_{0}$ on $O$. 
Then $A_{0}$ is the perverse extension of $\mathcal{L}_{0}$.

This construction suggest the following (seemingly) more general construction of parabolic character sheaves on $Z$. Let $O$ be any $B \times B$-orbit in $Z$ and let $A$ be the perverse extension of a suitable local system on $O$ that is equivariant for the $B_{d}$-action on $Z$. Define a perverse sheaf $\breve{A}$ on $G \times Z$ as before. Then define a parabolic character sheaf to be a perverse constituent of $q ! \breve{A}$, where $q: G \times{ }_{B} Z \rightarrow Z$. This definition was given (in an important special case) independently by myself and He, who proved in [H2] that no new character sheaves appear. We discuss now another approach to these results, using the Hecke algebra representations of [Sp1]. We first fix notations.

Let $h=\phi(1,1)$ the base point of $Z$. For $w \in W, x \in W^{B, Q}$ put $O_{w, x}=$ $(B \times B) .(w, x) . h$.

4.2. Lemma. (i) The $O_{w, x}$ are the $B \times B$-orbits in $Z$;

(ii) The isotropy group in $B \times B$ of $(\dot{w}, \dot{x} . h)$ is a semi-direct product of a connected unipotent group and the subgroup $\left(t,\left(x \sigma w^{-1}\right)(t)\right)$ of $T \times T$.

Proof. By Bruhat's lemma, $Z$ is the union of the sets $(B \times B) \cdot\left(w, w^{\prime}\right) \cdot h$ with $w, w^{\prime} \in W$. Writing $w^{\prime}=x y$ with $x \in W^{B, Q}, y \in W_{M}$ we have $\left(w, w^{\prime}\right) \cdot h=$ $\left(w \sigma^{-1}\left(y^{-1}\right), x\right) . h$ from which we see that $Z$ is the union of the $O_{w, x}$.

If $O_{w, x}=O_{w^{\prime}, x^{\prime}}$ then applying the projection $Z \rightarrow G / Q$ we see that $B x Q=$ $B x^{\prime} Q$, whence $x=x^{\prime}$. If $\left(b, b^{\prime}\right)(w, x)=\left(w^{\prime}, x\right)$ then $b w=w^{\prime} l u, b^{\prime} x=x \sigma(l) v$ with $l \in L, u \in U, v \in V$. Then $B x C=B x \sigma(l) C$ and by Bruhat's lemma $l=1$. It follows that $B w B=B w^{\prime} B$ and $w=w^{\prime}$. This proves (i). The proof of (ii) is left to the reader.

Put $V=W \times W^{B, Q}$, the set of $B \times B$-orbits in $Z$. Let $v=O_{w, x}$ and put $\dot{v}=(\dot{w}, \dot{x}) \cdot h$.

4.3. Lemma. (i) There is a unique morphism $\rho: v \rightarrow$ T such that $\left(u \dot{w} \rho(z), u^{\prime} \dot{x}\right) \cdot h=$ $z$ with $u, u^{\prime} \in U_{B}$, for all $z \in v$;

(ii) For $t, t^{\prime} \in T, u, u^{\prime} \in U_{B}$ we have $\rho\left(t u, t^{\prime} u^{\prime}\right) . z=w^{-1}(t)\left(\sigma^{-1} x^{-1}\right)\left(t^{\prime}\right)^{-1} \rho(z)$;

(iii) The isotropy group $(B \times B)_{i}$ is connected.

Proof. The existence of $t \in T$ with $z=\left(u \dot{w} t, u^{\prime} \dot{x}\right) . h$ is clear. If $t^{\prime} \in T$ has the same property then $t^{-1} t^{\prime}$ must be unipotent so that $t=t^{\prime}$. The existence of $\rho$ follows. The proofs of (ii) and (iii) are left to the reader. 
4.4. We perform constructions similar to those of 2.2. If $\xi \in \hat{X}(T)$ let $\mathcal{L}_{\xi, \dot{v}}=\rho^{*} \xi$, a rank one local system on $v$. The perverse sheaf $A=A_{\xi, \dot{w}}$ on $Z$ is the perverse extension of $\mathcal{L}_{\xi, \dot{v}}$. $A$ has weight $(w . \xi,-(x \sigma) . \xi)$ for the $B \times B$-action (in the sense of $[\mathrm{MS} 2,2.2])$.

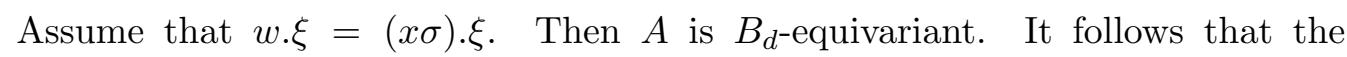
parabolic character sheaves are the irreducible constituents of the semisimple complexes $C_{\xi, \dot{v}}=q_{!} \breve{A}$ of 4.1 . We write $C_{\xi, v}$ if only its isomorphism class matters.

4.5. We first give some complements to the facts recalled in 1.7. The positive roots and the length function on $W$ are determined by $B$. We then have $P=P_{J}$ and $Q={ }^{a} P_{K}$, where $a \in W^{K}$. Then $\sigma$ is an isomorphism $L_{J} \rightarrow{ }^{y} L_{K}$, inducing a bijection $J \rightarrow a . K$, also denoted by $\sigma$. Now $W^{P, Q}=W^{J, K} a^{-1}$.

If $w \in W^{K}$ and $s \in I$ is a simple reflection then

$$
\text { either } s w \in W^{K} \text { or } w^{-1} s w \in K \text {. }
$$

Let $Y$ be the the group of cocharacters of $T$, viewed as the dual of $X=X(T)$ and put $\hat{Y}=\left(\mathbb{Z}_{(p)} / \mathbb{Z}\right) \otimes Y$. We have a pairing $\langle$,$\rangle between \hat{X}=\hat{X}(T)$ and $\hat{Y}$, with values in $\mathbb{Z}_{(p)} / \mathbb{Z}$. For $\alpha \in R$ the cocharacter $\alpha^{\vee}$ defines an element $\check{\alpha} \in \hat{Y}$.

4.6. We will use the generalized Hecke algebra $\mathcal{K}$ of $[\mathrm{MS} 2$, 4.3.2]. It is a free module over $R=\mathbb{Z}\left[u, u^{-1}\right]$ with basis $\left(e_{\xi, x}\right)$ where $x \in W$ and $\xi \in \hat{X}$.

The multiplication in $\mathcal{K}$ is described by the following rules:

(a) $e_{\xi, x} e_{\eta, y}=0$ if $\xi \neq y . \eta$.

(b) If $s$ is a simple reflection and $\xi=y . \eta$ this product equals

$$
\begin{array}{ll}
e_{\eta, s y} & \text { if } l(s y)>l(y), \\
\left(u^{2}-1\right) e_{\eta, y}+u^{2} e_{\eta, s y} & \text { if } l(s y)<l(y) \text { and }\langle y . \eta, \check{\alpha}\rangle=0, \\
u^{2} e_{\eta, s y} & \text { if } l(s y)<l(y) \text { and }\langle y . \eta, \check{\alpha}\rangle \neq 0 .
\end{array}
$$

Moreover, $e_{\xi, 1} e_{\eta, y}=\delta_{\xi, y . \eta} e_{\eta, y}$ and $e_{\xi, x} e_{\eta, 1}=\delta_{\xi, \eta} e_{\xi, x}$.

4.7. With a spherical variety there is associated in [MS2] a representation of $\mathcal{K}$. We describe it for the $G \times G$-spherical variety $Z$. The results are very similar 
to those discussed in [Sp1], for a slightly more restricted situation. The algebra for $G \times G$ is $\mathcal{K} \otimes_{R} \mathcal{K}$. We write $e_{\xi, x}, f_{\xi, x}$ for $e_{\xi, x} \otimes 1$, respectively, $1 \otimes e_{\xi, x}$.

Let $\mathcal{G}$ be the Grothendieck group of the abelian category of perverse sheaves on $Z$ and write $\mathcal{G}_{u}$ for the $\mathbb{Z}\left[u, u^{-1}\right]$-module $\mathbb{Z}\left[u, u^{-1}\right] \otimes \mathcal{G}$.

The module $\mathcal{M}$ is introduced along the lines of [MS2, Sect. 3]. It is contained in $\mathcal{G}_{u}$. In this setting $\mathcal{M}$ has a basis of "Kazhdan-Lusztig elements" $c_{\xi, v}=c_{\xi, w, x}$, where $c_{\xi, v}$ is the class of the perverse sheaf $A_{\xi, v}$ of 4.4. We shall also use the basis $\left(m_{\xi, v}\right)=\left(m_{\xi, w, x}\right)\left(\xi \in \hat{X}, w \in W, x \in W^{B, Q}\right)$ that is defined by the local system $\mathcal{L}_{\xi, v}$ (cf. [loc. cit.]).

We have $W^{B, Q}=W^{K} a^{-1}$. Put $x=\bar{x} a^{-1}$. By (11) either $s x \in W^{B, Q}$ or $s x=x \sigma(\tilde{s})$ where $\tilde{s} \in J$. We describe the $\mathcal{K} \otimes \mathcal{K}$-module structure of $\mathcal{M}$ in terms of the basis $\left(m_{\xi, w, x}\right)$.

4.8. Lemma. (i) $e_{\eta, s} m_{\xi, w, x}=0$ if $\eta \neq w . \xi$ and $e_{\eta, 1} m_{\xi, w, x}=\delta_{\eta, x . \xi} m_{\xi, w, x}$;

(ii) If $\eta=w . \xi$ the product $e_{\eta, s} m_{\xi, w, x}$ equals

(a) $m_{\xi, s w, x}$ if $l(s w)>l(w)$,

(b) $\left(u^{2}-1\right) m_{\xi, w, x}+u^{2} m_{\xi, s w, x}$ if $l(s w)<l(w)$ and $\langle w . \xi, \check{\alpha}\rangle=0$,

(c) $u^{2} m_{\xi, s w, x}$ if $l(s w)<l(w)$ and $\langle w . \xi, \check{\alpha}\rangle \neq 0$;

(iii) $f_{\eta, s} m_{\xi, w, x}=0$ if $\eta \neq(x \sigma) . \xi$ and $f_{\eta, 1} m_{\xi, w, x}=\delta_{\eta,(x \sigma) . \xi} m_{\xi, w, x}$;

(iv) If $\eta=(x \sigma) . \xi$ and $s x \in W^{B, Q}$ the product $f_{\eta, s} . m_{\xi, w, x}$ equals

(a) $m_{\xi, w, s x}$ if $l(s \bar{x})>l(\bar{x})$,

(b) $\left(u^{2}-1\right) m_{\xi, w, x}+u^{2} m_{\xi, w, s x}$ if $l(s \bar{x})<l(\bar{x})$ and $\langle(x \sigma) . \xi, \check{\alpha}\rangle=0$,

(c) $u^{2} m_{\xi, w, s \bar{x}}$ if $l(s \bar{x})<l(\bar{x})$ and $\langle(x \sigma) \cdot \xi, \check{\alpha}\rangle \neq 0$;

(v) If $\eta=(x \sigma) . \xi$ and $s x \notin W^{B, Q}$ the product of (iv) equals $m_{\xi, w \tilde{s}, x}$.

Proof. This follows from the formulas of [MS2, 4.3.1]. Cf. the proof of [Sp1, Lemma 5.2].

4.9. If $A=\oplus_{i} A_{i}\left[n_{i}\right]$ is a semisimple complex on $Z$ (the $A_{i}$ being perverse) define $\chi(A) \in \mathcal{G}_{u}$ by

$$
\chi(A)=\sum_{i} u^{-n_{i}}\left[A_{i}\right]
$$

Let $v=O_{w, x}$ be a $B \times B$-orbit on $Z$ and let $\xi \in \hat{X}(T)$ be such that $w \cdot \xi=(x \sigma) . \xi$. We then have the semisimple complex $C_{\xi, v}$. Let $h$ be the $\mathbb{Z}\left[u, u^{-1}\right]$-linear map 
$\mathcal{M} \rightarrow \mathcal{G}_{u}$ with

$$
h\left(c_{\xi, w, x}\right)=\chi\left(C_{\xi, v}\right) .
$$

4.10. Lemma. For $\eta \in \hat{X}(T), s \in I, m \in \mathcal{M}$ we have $h\left(e_{\eta, s} . m\right)=h\left(f_{\eta, s} . m\right)$. Proof. For $s \in I$ let $P_{s} \supset B$ be the corresponding minimal parabolic subgroup. If $A$ is a $B \times B$-weighted perverse sheaf on $Z$ (a perverse sheaf with a weight for the $B \times B$-action) the product $e_{\eta, s} \cdot[A] \in \mathcal{M}$ is defined as follows, see [MS2, Sect. 3]. There is a $B \times B$-weighted perverse sheaf $A_{\eta}$ on $P_{s}$ such that $A_{\eta} \otimes A$ is, up to a shift, the pull-back of a perverse sheaf $\tilde{A}$ on the quotient $P_{s} \times{ }^{B} Z$ of $P_{s} \times Z$ by the $B$-action $b .(p, z)=\left(p b^{-1},(b, 1) . z\right)$. Let $\alpha$ be the proper morphism $P_{s} \times^{B} Z \rightarrow Z$ induced by $(p, z) \mapsto(p, 1) . z$. Then $\alpha ! A$ is a semisimple complex $\oplus_{i} A_{i}\left[n_{i}\right]$ and $e_{\eta, s}[A]=\sum u^{-n_{i}}\left[A_{i}\right]$.

If now $A_{i}=A_{\xi_{i}, v_{i}}$ we have

$$
h\left(e_{\eta, s} \cdot[A]\right)=\sum_{i} u^{-n_{i}} \chi\left(C_{\xi_{i}, v_{i}}\right),
$$

notations being as in 4.4. Putting together the construction of the multiplication by $e_{\eta, s}$ and the construction of 4.4 we are led to considering the morphism

$$
G \times P_{s} \times Z \rightarrow Z
$$

sending $(g, p, z)$ to $(g p, g) . z$. The group $B \times B$ operates on $G \times P_{s} \times Z$ by

$$
\left(b, b^{\prime}\right) \cdot(g, p, z)=\left(g b^{-1}, b p\left(b^{\prime}\right)^{-1},\left(b^{\prime}, b\right) \cdot z\right) .
$$

The quotient $Z_{1}$ of $G \times P_{s} \times Z$ for the $B \times B$-action exists and $\alpha$ defines a morphism $\alpha_{1}: Z_{1} \rightarrow Z$. There is a perverse sheaf $A_{1}$ on $Z_{1}$ such that

$$
h\left(e_{\eta, s} . c_{\xi, v}\right)=\left[\left(\alpha_{1}\right) ! A_{1}\right] .
$$

The pull-back of $A_{1}$ to $G \times P_{s} \times Z$ is, up to a shift, $E \otimes A_{\eta} \otimes A_{\xi, O_{w, x}}$.

On the other hand, proceeding similarly with the morphism $G \times P_{s} \times Z \rightarrow Z$ sending $(g, p, z)$ to $(g, g p) . z$ we obtain a morphism $\beta_{1}: Z_{1} \rightarrow Z$ with $h\left(f_{\eta, s} \cdot c_{\xi, v}\right)=$ $\left[\left(\beta_{1}\right) ! A_{1}\right]$. Let $\gamma$ be the automorphism of $Z_{1}$ induced by the automorphism $(g, p, z) \mapsto\left(g p, p^{-1} z\right)$ of $G \times P_{s} \times Z$. Then $\beta_{1}=\alpha_{1} \circ \gamma$ and $\left(\beta_{1}\right) !=\left(\alpha_{!}\right) ! \circ \gamma_{!}$. Since $A_{1}$ and $\gamma_{!} A_{1}$ are isomorphic so are $\left(\alpha_{1}\right) ! A_{1}$ and $\left(\beta_{1}\right) ! A_{1}$. The Lemma follows. 
The next result is more or less equivalent with the "key lemma" 3.12 of [H3].

4.11. Proposition.. $h\left(m_{\xi . w, x}\right) \in \mathcal{G}_{t}\left(\xi \in \hat{X}, w \in W, x \in W^{B, Q}\right)$ is a linear combination of the special elements $h\left(m_{\xi, 1, y}\right)\left(y \in W^{B, Q}\right)$.

Proof. Use induction on $l(w)$, starting with $w=1$. Assume that $l(w)>0$ and write $w=s z$ with $s \in I, l(z)=l(w)-1$. By part (ii)(a) of Lemma 4.8 and Lemma 4.10

$$
h\left(m_{\xi, w, x}\right)=h\left(f_{z \cdot \xi} \cdot m_{\xi, z, x}\right) .
$$

By Lemma 4.8 (iii) the right-hand side is 0 unless $z \cdot \xi=(x \sigma) . \xi$. If we are not in case (v) of Lemma 4.8 it is a linear combination of elements $h\left(m_{\xi, z, x^{\prime}}\right)$ and induction can be invoked.

In the exceptional case we have $x^{-1} s x=\sigma(\tilde{s}) \in K$ and $w=s z=z \tilde{s}$. Let $w=s_{1} s_{2} \ldots s_{l}$ be a reduced decomposition with $s_{1}=s$. Then $w=s_{2} \ldots s_{l} \tilde{s_{1}}$ is a second reduced decomposition. Iterating the argument we see that if $x^{-1} s_{i} x \notin K$ for some $i$ we can again invoke induction. If however $x^{-1} s_{i} x \in K$ for all $i$, the argument shows that $w=\sigma^{-1}\left(x^{-1} w x\right)$, showing that $w \in W_{J}$.

Since the elements $(l, \sigma l)(l \in L)$ fix $h$

$$
(B \times B)(w, x) \cdot h=(B \times B)\left(1, x(\sigma w)^{-1}\right) \cdot h=(B \times B) \cdot\left(1, w^{-1} x\right) . h .
$$

As in 3.6 let $Z_{0}$ be the image in $Z$ of $\{1\} \times G$ and let $p: G \rightarrow Z_{0}$, a fibration with fiber $V$. Then

$$
O_{w, x}=(B \times B)(w, x) \cdot h=p\left(B w^{-1} x C\right) .
$$

Notice that under our assumptions

$$
w^{-1} x \sigma \cdot \xi=\xi
$$

Also, the closure $\overline{O_{w, x}}$ is a union of some $p\left(B w^{\prime} C\right)$. It follows that $h\left(c_{\xi, w, x}\right)$ is a linear combination of classes $[A]$ where $A$ is an irreducible constituent of a semisimple complex $C_{\xi, w^{-1} x}$ of 2.2 and a similar property holds for the $h\left(c_{\xi^{\prime}, w^{\prime}, x^{\prime}}\right)$ where $O_{w^{\prime}, x^{\prime}} \subset \overline{O_{w, x}}$. Notice that $h\left(m_{\xi, w, x}\right)$ is a linear combination of such $h\left(c_{\xi^{\prime}, w^{\prime}, x^{\prime}}\right)$.

By Lemma 2.3 and the observations of 4.1 and 2.9 the isomorphism classes of the constituents of $C_{\xi, w^{-1} x}$ are already obtained from the complexes with $w=1$. This means that our $h\left(c_{\xi, w, x}\right)$ is a linear combination of elements $h\left(c_{\xi^{\prime}, 1, x^{\prime}}\right)$. Then 
$h\left(c_{\xi, w, x}\right)$ is a linear combination of certain $h\left(m_{\xi^{\prime}, 1, x^{\prime}}\right)$. The Proposition follows.

4.12. Corollary. The parabolic character sheaves of 4.4. coincide with those of 3.8.

The Corollary was first proved by He in [H2].

\section{The compactification of $G$.}

\subsection{The group $\bar{\Delta}$.}

This Section is somewhat sketchy. Notations being as in 1.8, let $\bar{L}$ and $\bar{M}$ be the adjoint groups of $L$ and $M$ and let $\bar{\sigma}$ be the isomorphism $\bar{L} \rightarrow \bar{M}$ induced by $\sigma$. Denote by $\bar{\pi}_{P}$ and $\bar{\pi}_{Q}$ the projections $P \rightarrow \bar{L}, Q \rightarrow \bar{M}$ and put

$$
\bar{\Delta}=\bar{\Delta}_{G, P, Q, \bar{\sigma}}=\left\{(p, q) \in P \times Q \mid \bar{\pi}_{Q}(q)=\bar{\sigma}(\bar{\pi}(p))\right\} .
$$

There is an obvious homomorphism $\Delta \rightarrow \bar{\Delta}$ whose kernel is isomorphic to the center $C$ of $L$.

From now on assume $G$ to be adjoint. Then $C$ is a torus. It acts on the right on $G \times G$ by $c .(x, y)=(x c, y(\sigma c))$. This induces a right action of the torus $C$ on $Z$. We view $\bar{Z}$ as the quotient $Z / C$. Let $\pi: Z \rightarrow \bar{Z}$ be the canonical map, a torus fibration.

5.2. Lemma. An irreducible perverse sheaf $A$ on $Z$ is of the form $\pi^{*}(\bar{A})[\operatorname{dim} C]$ for some perverse sheaf $\bar{A}$ on $\bar{Z}$ if and only if it is $C$-equivariant.

A parabolic character sheaf $\bar{A}$ on $\bar{Z}$ is an irreducible perverse sheaf obtained as in the Lemma from a $C$-equivariant parabolic character sheaf $A$ on $Z$. Then $A$ is an irreducible constituent of a complex $C_{\xi, v}$ as in 4.4, where the restriction of $\xi$ to $C$ is zero.

Applying Prop.1.6 (i) we associate to a parabolic character sheaf on $\bar{Z}$ an irreducible perverse sheaf on $G$, which we call a $\bar{\Delta}$-character sheaf on $G$.

5.3. Lemma. The $\bar{\Delta}$-character sheaves on $G$ are the $\Delta$-character sheaves that are equivariant for the action of $C$ on $G$ by left multiplication. 
5.4. With the notations of 1.7 let $B$ be the Borel group $P_{\emptyset}$. If $P_{J}$ is a standard parabolic subgroup denote by $P_{J}^{-}$the opposite parabolic subgroup. It shares the Levi subgroup $L_{J}$ with $P_{J}$. Put

$$
\bar{\Delta}_{J}=\bar{\Delta}_{G, P_{J}^{-}, P_{J}, \mathrm{id}}, \bar{Z}_{J}=G \times G / \bar{\Delta}_{J} .
$$

Let $X$ be the wonderful compactification of our adjoint group $G$. The group $G \times G$ acts on it with finitely many orbits. These orbits are precisely our $\bar{Z}_{J}$.

A parabolic character sheaf $A$ on $\bar{Z}_{J}$ extends canonically to an irreducible perverse sheaf $\tilde{A}$ on the closure

$$
\operatorname{cl}\left(\bar{Z}_{J}\right)=\bigcup_{K \subset J} \bar{Z}_{K}
$$

The restriction of $\tilde{A}$ to $\bar{Z}_{K}(K \subset J)$ is a complex which one would like to know more about.

We can transfer things to $G$ by applying Prop. 1.6 (i) (or rather a version of it for complexes in $\mathcal{D}(G)$ ). We then see that a $\bar{\Delta}_{J}$-character sheaf $A$ determines for each $K \subset J$ a $\bar{\Delta}_{K}$-equivariant complex $A_{K}$ on $G$.

5.5. Question. Can $A_{K}$ be described in terms of a restriction operation on $\Delta_{J}$-equivariant complexes on $G$, for varying $J$ ?

\section{References}

[C] R. W. Carter, Finite groups of Lie type. Conjugacy classes and complex characters, Wiley, 1985

[EL] S. Evens and J.-H. Lu, On the variety of Lagrangian subalgebras, II, Ann. Sci. Éc. Norm. Sup. 39 (2006), 347-379

[H1] X. He, Unipotent variety in the group compactification, Adv. in Math. 203 (2006), 103-131

[H2] X. He, The $G$-stable pieces of the wonderful compactification, Trans. Amer. Math. Soc. 359 (2007), 3005-3024 
[H3] X. He, The character sheaves on the group compactification, Adv. in Math. 207 (2006), 805-827

[L1] G. Lusztig, Character sheaves, III, Adv. Math. 57 (1985), 266-315

[L2] G. Lusztig, Parabolic character sheaves I, Moscow Math. J. 4 (2004), 153-179; II, ibid., 869-896

[L3] G. Lusztig, A class of perverse sheaves on a partial flag manifold, Repr. Theory 11 (2007), 122-171

[MS1] J. G. M. Mars and T. A. Springer, Character sheaves, Astérisque 173174 (1988), 111-198

[MS2] J. G. M. Mars and T. A. Springer, Hecke algebra representations related to spherical varieties, J. Repr. Theory, 11 (1998), 33-69

[Sp1] T. A. Springer, Intersection cohomology of $B \times B$-orbit closures in group compactifications, J. Alg. 258 (2002), 71-111

[Sp2] T. A. Springer, An extension of Bruhat's lemma, J. Alg. 313 (2007), $417-427$

T. A. Springer

Mathematisch Instituut

Budapestlaan 6, 3584 CD Utrecht

the Netherlands

Email: T.A.Springer@uu.nl 